
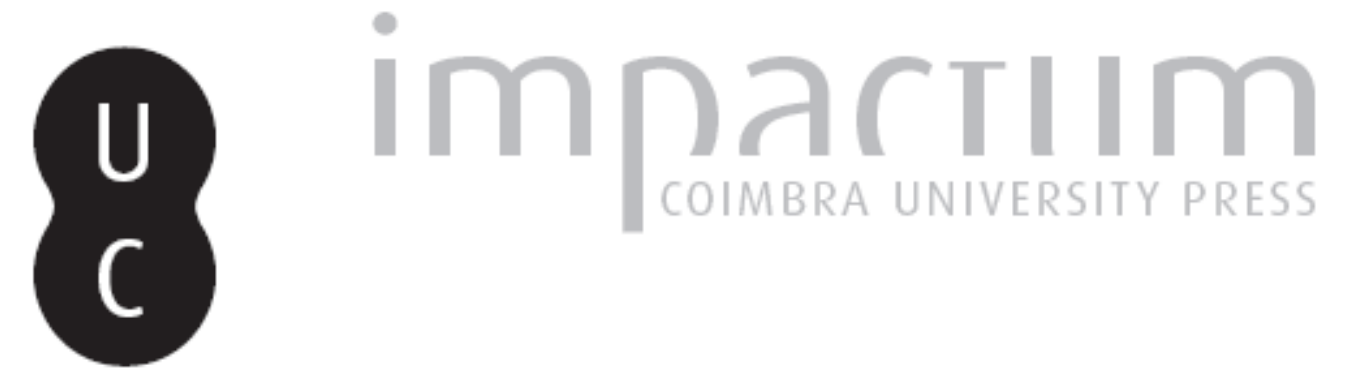

\title{
Nuove motivazioni e pubblici della lingua italiana nel mondo
}

Autor(es): Vedovelli, Massimo

Publicado por: Imprensa da Universidade de Coimbra

URL persistente:

URl:http://hdl.handle.net/10316.2/42700

DOI:

DOI:https://doi.org/10.14195/0870-8584_1_15

Accessed : $\quad$ 26-Apr-2023 11:07:02

A navegação consulta e descarregamento dos títulos inseridos nas Bibliotecas Digitais UC Digitalis, UC Pombalina e UC Impactum, pressupõem a aceitação plena e sem reservas dos Termos e Condições de Uso destas Bibliotecas Digitais, disponíveis em https://digitalis.uc.pt/pt-pt/termos.

Conforme exposto nos referidos Termos e Condições de Uso, o descarregamento de títulos de acesso restrito requer uma licença válida de autorização devendo o utilizador aceder ao(s) documento(s) a partir de um endereço de IP da instituição detentora da supramencionada licença.

Ao utilizador é apenas permitido o descarregamento para uso pessoal, pelo que o emprego do(s) título(s) descarregado(s) para outro fim, designadamente comercial, carece de autorização do respetivo autor ou editor da obra.

Na medida em que todas as obras da UC Digitalis se encontram protegidas pelo Código do Direito de Autor e Direitos Conexos e demais legislação aplicável, toda a cópia, parcial ou total, deste documento, nos casos em que é legalmente admitida, deverá conter ou fazer-se acompanhar por este aviso.

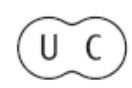




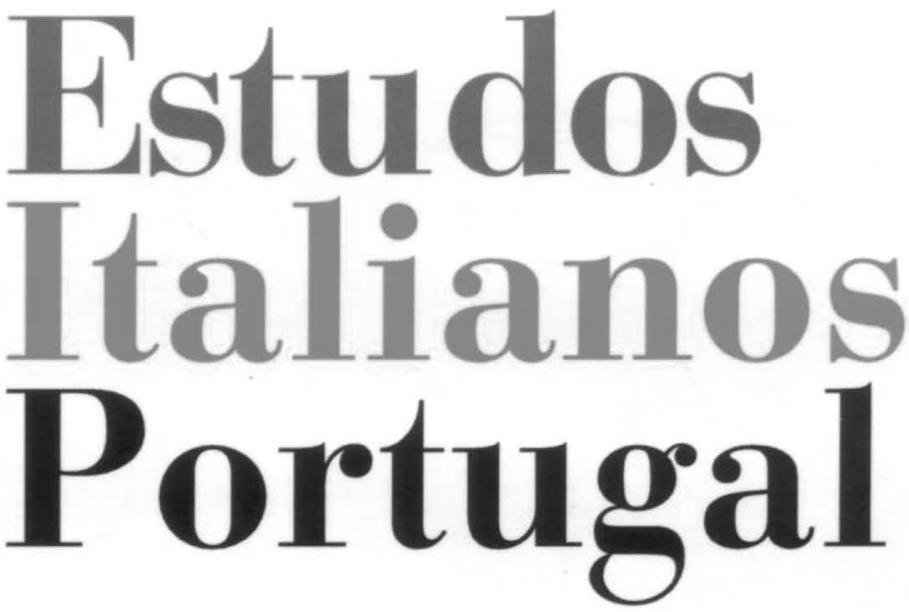

Instituto

Italiano

de Cultura

em Portugal

Nova Série

$\mathbf{N}^{\circ} \mathbf{1}$

2006 
NUOVE MOTIVAZIONI E PUBBLICI DELLA

LINGUA ITALIANA NEL MONDO

Massimo Vedovelli

\section{Obiettivi}

Non è infrequente, almeno in Italia, il dibattito sulla condizione della nostra lingua diffusa nel mondo: si tratta di una materia che sempre più spesso appare sfuggire dal campo di interesse dei ricercatori e di quanti se ne occupano per professione (ad esempio, insegnanti, autori di materiali didattici, dirigenti di istituzioni formative), per vedere gli interventi dei non specialisti, fra i quali si possono annoverare anche diversi politici e giornalisti. Lanciata nella dimensione della comunicazione di massa, la questione dello stato di salute della lingua italiana nel mondo assume forme troppo nette e contrapposte per poter rispondere pienamente alla complessità della situazione, almeno così come essa si manifesta a chi se ne occupa con intento scientifico o didattico. Ecco, allora, che ai non specialisti la questione della lingua assume ora $\mathrm{i}$ toni trionfalistici di chi si inorgoglisce per il fatto che l'italiano è uno dei primi quattro o cinque idiomi più studiati nel mondo come lingua straniera, o chi lo vede essere onnipresente - subito dopo l'inglese - nella comunicazione sociale a livello planetario: quella, per intendersi, fatta da insegne di negozi e manifesti pubblicitari ${ }^{1}$. Dall'altro

${ }^{1}$ Su tale argomento ved. Vedovelli (2005). 
versante rispondono coloro che vedono la situazione dell'italiano ormai totalmente perdente nel consesso mondiale delle lingue, e totalmente marginalizzata a livello di uso nelle istituzioni comunitarie europee. A ciò costoro aggiungono una analisi pessimista sulla condizione dell'italiano in Italia, soprattutto fra le giovani generazioni.

Per superare i limiti di una analisi che si nutra solo delle polemiche contrapposte e superficialmente alimentate, spesso ingigantite dalla stampa alla ricerca di notizie ad effetto, sembra necessario recuperare il riferimento ai dati di fatto per poter tentare interpretazioni che siano fondate e capaci di orientare azioni adeguate di diffusione del nostro idioma. Proprio per raggiungere questo obiettivo, sintetizziamo i risultati dell'indagine Italiano 2000, promossa dal Ministero degli Affari Esteri, Direzione Generale per la Promozione e la Cooperazione Culturale, sui pubblici stranieri che nel mondo si avvicinano alla nostra lingua rendendola oggetto di apprendimento. L'indagine è stata affidata al Dipartimento di Studi Linguistici e Letterari dell'Università 'La Sapienza' di Roma, ed è stata diretta dal prof. Tullio De Mauro, il quale, assunte cariche di governo, si è avvalso della collaborazione dello scrivente, della dott.ssa Monica Barni e del dott. Lorenzo Miraglia, operanti presso l'Università per Stranieri Siena. L'indagine risale al 2000, ed è l'ultima su grande scala realizzata sull'argomento. Essa ha avuto come obiettivo primario le dinamiche della diffusione dell'italiano all'estero, ma anche l'analisi della sua posizione entro le nostre comunità all'estero. Non dimentichiamo, infatti, che la lingua italiana come lingua d'uso è una realtà idiomatica abbastanza recente, e che i nostri emigrati, soprattutto (ma non solo) quelli della grande ondata migratoria della seconda metà del XIX secolo, sono andati all'estero portando con sé quasi esclusivamente una competenza dialettale: l'italiano si è diffuso nel mondo, ma con ciò dobbiamo intendere anche la sua diffusione entro le nostre comunità emigrate.

\section{I MOTIVI DELL'INDAGINE ITALIANO 2000}

Nell'esaminare la condizione dell'italiano fuori dei confini nazionali è necessario tenere presente il momento di svolta che a livello planetario sta vivendo il sistema di diffusione delle lingue e dei corrispettivi sistemi sociali. Si parla di un nuovo ordine linguistico mondiale perché, ridisegnandosi i confini delle identità nazionali e delle istituzioni sopranazionali, si è arrivati a una nuova gerarchizzazione dei rapporti fra le lingue. Tale ordine è costituito da sistemi in cui una società si lega inscindibilmente alle sue manifestazioni culturali e linguistiche, diventando soggetto capace di promuovere o proporre modelli di identità, oppure destinatario delle proposte di altri soggetti o comunque soggetto sollecitato a misurare la propria identità con quella dei modelli che si espandono in maniera predominante a livello planetario. In questo sistema la posizione dell'italiano, inteso come nesso di identità linguistica e culturale, appare particolarmente delicata perché al centro di spinte contrapposte: la grande ricchezza in termini di capacità di proposta, ma anche i molti elementi problematici.

La relativa novità della lingua d'uso unitariamente diffusa; l'identità giocata fra il retaggio di un'alta tradizione intellettuale e le spinte innovative verso nuovi codici che si manifestano nei vari piani della società nazionale; l'immagine internazionale dell'Italia legata a tradizionali stereotipi e insieme capace di produrre attese di innovatività e di creatività continua; il rinnovamento del sistema produttivo e la posizione dell'Italia fra i paesi più industrializzati: tutti questi aspetti si riflettono nella generale identità idiomatica della nostra società costituendo le condizioni di possibilità della sua capacità di proporsi in una posizione non secondaria né marginale entro il sistema mondiale delle società e delle loro lingue. 


\section{La situazione attuale dell'italiano Nel Sistema} DELLE LINGUE: CORRELATI SOCIALI, ECONOMICI, CULTURALI

Ciò che ha spinto all'indagine Italiano 2000 è un insieme di indizi, di dati parziali, di elementi che, sia pur non sistematicamente interrelati fra di loro a costituire un tutto coerente, concorrono a delineare l'idea di una realtà profondamente mutata dell'italiano diffuso fra stranieri al di fuori dei confini nazionali. L'idea che muove alla necessità d'una possibile ricerca non è tanto la consapevolezza di tale mutamento in alcune realtà quali, ad esempio, quelle dove la presenza storica delle nostre comunità emigrate ha comportato l'attivazione sistematica dei corsi di lingua-cultura, quanto il fatto che anche la più generale progettazione degli interventi linguistico-culturali, quali che siano i loro destinatari, deve porsi in sistematica sintonia con un quadro strutturale generale nel quale non solo la dimensione linguistica, ma anche quella sociale, economico-produttiva e istituzionale concorrono a formare un sistema le cui componenti definiscono valori e sensi in un gioco reciproco di forze, tensioni, spinte propulsive. Dire che negli ultimi dieci anni sono cambiati i pubblici dell'italiano lingua straniera (d'ora in poi useremo, seguendo Giacalone Ramat, 1986, l'iperonimo L2), cioè gli stranieri che si avvicinano alla nostra lingua in un contesto di contatto e di apprendimento guidato e/o spontaneo, rappresenta una verità che riguarda innanzitutto i pubblici adulti, quelli più frequentemente oggetto delle varie indagini finora svolte, in maniera più o meno sistematica, a partire da quella ancora fondamentale diretta da Ignazio Baldelli alla fine degli anni '70 per conto dell' Istituto per l'Enciclopedia Italiana, su incarico del Ministero degli Affari Esteri (Baldelli, 1987).

Ciò che oggi possiamo dire, rileggendo quella indagine presentata al grande convegno del 1982 sull'italiano nel mondo, è che essa manifesta uno scarto fra la dimensione descrittiva e quella interpretativa: le conclusioni alle quali giunse oggi appaiono caratterizzate in maniera ampiamente diversa proprio a causa dell'agire di una serie di condizioni che hanno mutato, proprio a partire dalla fine degli anni Settanta, il quadro nel quale andava a diffondersi l'italiano ${ }^{2}$. Da quegli anni, infatti, il nesso lingua-cultura-società italiana ha sviluppato la sua capacità di attrazione presso gli stranieri ed è diventato oggetto di interesse entro un sistema planetario che oggi appare radicalmente cambiato e che proprio nella seconda metà di quel decennio stava innescando un mutamento epocale a tutti i suoi livelli.

Il rischio di considerare solo questa dimensione dell'oggetto di studio sta in una conseguenza che può derivarne, ovvero il fatto che la lingua italiana possa vivere di una 'rendita di capitale' capace di giustificare l'assenza di sistematici interventi in termini di politica di diffusione linguistica. Alla base di tale impostazione c'è l'idea che il legame fra la lingua e la tradizione intellettuale italiana connoti in termini esclusivamente culturali le motivazioni degli stranieri al contatto, allo studio, all'apprendimento della nostra lingua. Dunque, la formidabile eredità intellettuale italiana nei settori della letteratura, dell'arte, della musica, e lo spessore delle vicende storiche che la contestualizzano costituiscono un capitale sociale per il nesso lingua-cultura-società italiana sul quale si potrà sempre contare, e che rappresenta perciò la giustificazione alla identificazione di altre urgenze nel momento in cui lo Stato guarda da una prospettiva linguistica oltre i propri confini politici.

2 Il convegno fu organizzato dalla Presidenza del Consiglio dei Ministri e dal Ministero degli Affari Esteri. Per gli atti del convegno ved. Presidenza del Consiglio dei Ministri (1983); per la presentazione dei risultati dell'indagine ved. Baldelli (1987); per una discussione sulla sua rilevanza nello sviluppo della politica linguistica per l'italiano L2 cfr. Vedovelli 2002 a, b). 
La rendita di capitale costituita dal legame fra lingua italiana e identità intellettuale si collega a un altro punto fermo del quadro entro il quale si è sempre situata la politica statale su tali tematiche, ovvero il presupposto della dimensione elitaria, del numero limitato di stranieri caratterizzati dall'ambizione di studiare la nostra lingua: in questa visione l'italiano è considerato, allora, una lingua di cultura e di pochi. In un momento storico in cui la politica coloniale dei grandi Stati si corroborava anche di una politica di diffusione della propria lingua, come avviene per l'inglese, che dal 1913 si dota di una certificazione di competenza linguistica, l'Italia sceglie invece di non puntare all'allargamento della base di stranieri fra i quali espandere l'italiano.

Con stupore e sorpresa furono accolti, all'inizio degli anni '80, i risultati dell'indagine sulla natura e sul destino dell'italiano come L2 per la novità del vedere la lingua italiana oggetto di forte attenzione a livello internazionale si accompagnava alla conferma che ciò era dovuto alla sua identità culturale 'alta', o meglio: intellettuale. Da un lato, perciò, una novità, e dall'altro una conferma. In più, l'idea che il solo destino della lingua italiana diffusa fra stranieri fosse legato al suo patrimonio culturale, al fatto che essa era la forma costituente di una storia culturale capace ancora di attrarre gli stranieri. Se questi si rivolgevano in numero sempre maggiore alla nostra lingua, ciò era dovuto alla capacità di prolungare il felice legame lingua - cultura intellettuale anche in altri fenomeni, che riposizionavano a livello internazionale la società italiana in quegli anni: l'industrializzazione, il boom economico, la fortuna di manifestazioni produttive legate a una base estetica (il design, la moda), l'immagine di una felice sintesi fra modernità, tradizione culturale, creatività; e ancora, la pluralità delle identità e la ricchezza delle tradizioni antropologiche. Tutti questi aspetti nuovi apparivano confermare e continuare, però, l'identità storicamente definita che legava la lingua italiana alle vette della cultura letteraria, artistica, musicale e delle altre manifestazioni della produzione intellettuale.

Ciò che è scaturito dall'indagine della fine degli '70 è noto, e non è questa la sede per riproporlo. Vogliamo solo ricordare, però, che nuove realtà planetarie stavano investendo l'Italia e la sua lingua, così come il sistema dei rapporti fra le lingue a diffusione internazionale. Proprio alla fine degli anni '70 viene prodotto il primo saggio sociologico su un fenomeno nuovo, allora, cioè l'immigrazione straniera in Italia; è del 1981 il primo articolo sui problemi linguistici degli immigrati stranieri ${ }^{3}$. Oggi ci è sempre più chiaro il fatto che l'italiano è lingua di contatto per almeno 2.600.000 immigrati stranieri adulti e bambini (stando ai recenti dati del Dossier Caritas), che apprendono e usano la nostra lingua non per interesse culturale né per turismo, ma per sopravvivere e vivere, per inserirsi socialmente e professionalmente nella società. E ancora, la nuova e consolidata posizione internazionale dell'Italia come paese industriale ha promosso l'attenzione degli stranieri anche per la nostra lingua come idioma capace di permettere scambi comunicativi nei settori della produzione e più generalmente dell'economia. Infine, la stessa composizione dei pubblici interessati culturalmente appare mutata, dal momento che si è passati da un generico interesse verso una tradizionale (se non stereotipata) immagine dell'identità culturale italiana allo specifico interesse degli studenti e dei docenti impegnati nei progetti di mobilità internazionale ${ }^{4}$.

\footnotetext{
3 Esperienze e proposte (1979); Vedovelli (1981).

${ }^{4}$ Per le indagini sui pubblici dell'italiano ved. Maggini / Parigi (1983), Covino Bisaccia (1989 e 1990), Bandini / Barni / Sprugnoli (1999), Vedovelli (1999), (2000), De Mauro / Vedovelli (1996).
} 
4. Le DisOMOgENEE SITUAZIONI DI DIFFUSIONE DELL'ITALIANO ALL'ESTERO: UN APPROCCIO SISTEMICO ALLA LORO ANALISI

L'indagine della fine degli anni Settanta, oltre a mettere in evidenza l'assoluta prevalenza di un'unica spinta motivazionale allo studio dell'italiano, ovvero quella del legame con la tradizione intellettuale, mette anche in evidenza alcuni elementi di cambiamento che solo in questi anni recenti si sono manifestati pienamente, e che la nuova indagine Italiano 2000 ha descritto. Nell'attuale quadro mondiale dei rapporti sociali e culturali fra i paesi e i popoli, la situazione dell'italiano sembra caratterizzata dalla disomogeneità, dalla compresenza contraddittoria di fattori contrastanti, dalla esistenza di punte avanzate di elaborazione sociale, produttiva, culturale e linguistica accanto a elementi tradizionali, sul piano linguistico-culturale. Questa doppia anima che attraversa l'intero sistema dell'italiano da un lato rappresenta un elemento di forza, di propulsione, una ricchezza e un patrimonio capace di proporre l'italiano in posizione non marginale nel mercato delle lingue; ma dall'altro rappresenta un elemento di stasi, di freno, che contribuisce a mantenere ancora viva l'immagine dell'Italia come paese di emigranti con la valigia di cartone. Le due anime convivono. Se ad esse e alle corrispondenti identità socioculturali e linguistiche si collegano le caratteristiche dell'azione istituzionale e del sistema non istituzionale delle agenzie formative che insistono nel settore dell'emigrazione italiana all'estero, ne deriva un quadro altamente disomogeneo. Già nell'indagine di Baldelli emergevano elementi di novità che sembravano preludere alla dinamizzazione della condizione dell'italiano degli stranieri, con la conseguenza, però, di produrre anche spinte contraddittorie.

Tale situazione, a volte contraddittoria, sempre ricca di elementi di novità, sottolinea la necessità di un approccio sistemico, strutturale nel momento in cui, come con Italiano 2000, la si vuole descrivere e interpretare nelle cause e negli effetti. A livello di metodologia della ricerca scientifica nel settore linguistico, ciò ha comportato elaborare una ipotesi di indagine che implicasse la definizione dei collegamenti fra i vari segmenti dei pubblici dell'italiano. Nel nostro caso si è trattato innanzitutto di collegare il pubblico reale e quello potenziale dei vari soggetti promotori di iniziative di formazione linguistica entro il sistema dell'italiano diffuso fra stranieri. Tale prospettiva si appoggia sull'idea che il sistema dell'italiano diffuso fra stranieri fuori dei confini nazionali è cambiato dagli anni '70 ad oggi quanto a condizioni socioculturali per la sua diffusione, soprattutto per ciò che riguarda il ruolo dell'Italia (come sistema sociale, produttivo, culturale, linguistico) entro il panorama internazionale. Tali cambiamenti hanno riguardato la composizione socioculturale, professionale, motivazionale dei pubblici tradizionali dell'italiano; hanno investito la politica delle istituzioni culturali italiane all'estero e la rete delle agenzie private e pubbliche della formazione linguistica. Ne sono state coinvolte le Università straniere e la politica dei governi che hanno inserito l'italiano entro i sistemi scolastici dei loro paesi o da essi l'hanno tolto. L'ipotesi generale che ha guidato Italiano 2000 è che i profondi cambiamenti a livello economico collettivo, sociale, culturale, di immagine del sistema Italia si sono riflessi sistematicamente in modo esteso su tutto il mercato dell'italiano L2 inteso come componente del generale mercato internazionale delle lingue.

È in tale quadro che è maturata l'esigenza di un'indagine conoscitiva in grado di mettere in luce eventuali cambiamenti circa i bisogni, le motivazioni, le esigenze di formazione linguistica, le caratteristiche socioculturali e linguistiche dei pubblici reali e potenziali dell'italiano L2 e della sua posizione nel mondo. 


\section{I RISUlTATI DI ITALIANO 2000}

Per il tipo di dati raccolti, il primo riferimento è costituito da una ricognizione effettuata dal Ministero degli Affari Esteri nel 1995 fra gli Istituti Italiani di Cultura (Ministero degli Affari Esteri, 1996): nonostante che Italiano 2000 abbia raccolto una notevole mole di dati presso un ventaglio molto diversificato di istituzioni e agenzie impegnate nella diffusione dell'italiano nel mondo, preferiamo limitarci a presentare in questa sede solo i dati che possono consentire il confronto con quelli citati: in tal modo restringiamo il campo dell'analisi ai soli soggetti istituzionali dipendenti dallo Stato italiano e, fra questi, solo gli Istituti di Cultura. In tal modo, pur se si perde la capacità di ricognizione globale, si guadagna in possibilità di confronto puntuale fra due situazioni relativamente distanti nel tempo. La validità dei dati è garantita, comunque, dalla rilevanza della posizione degli Istituti entro la rete dei soggetti impegnati nel settore dell'italiano L2: oltre a poter analizzare i cambiamenti che specificamente hanno riguardato gli Istituti, è possibile attribuire ai dati esaminati un valore più ampio, ovvero quello di indicatori dello stato generale dell'italiano nel mondo. I dati derivanti dagli altri soggetti informanti, infatti, mostrano le stesse linee di tendenza di quelle mostrate nella rete degli Istituti (d'ora in poi denominati $I I C)$.

\subsection{AmpieZZA DEL CORPUS DI INFORMANTI}

Hanno risposto al questionario 63 IIC sui 90 ai quali era stato inviato, corrispondenti al 70\% del totale. Da 15 IIC, il $16,7 \%$ del totale, non è pervenuto nessun tipo di informazione sui corsi: alcuni fra questi ultimi hanno comunicato la loro impossibilità a rispondere al questionario adducendo motivi che vanno dalla mancanza di personale capace di adempiere a questo compito a un consapevole e motivato rifiuto a svolgerlo. 12 IIC, il 13,3\% del totale, hanno comunicato che non organizzavano corsi di italiano negli anni 1999 o 2000.

Al di là della necessità metodologica di dare conto dell'ampiezza del corpus di informanti, la difformità che si manifesta entro questi dati segnala il tipo di condizione in cui operano gli IIC e la diversità di politiche attuate in sede locale nei confronti dell'offerta formativa di lingua italiana.

Altri 12 IIC, il 13,3\% del totale, hanno comunicato che non organizzano corsi di italiano. I dati quantitativi sono la manifestazione delle situazioni in cui gli IIC operano. Le caratteristiche sociali, economiche, culturali dei territori sui quali insistono gli IIC si concretizzano in cifre che globalmente, attraverso gli indicatori ai quali si riferiscono i dati, rimandano al complesso gioco dei fattori che caratterizzano le condizioni entro i quali si trovano ad operare gli IIC. In questo senso, le cifre e le interpretazioni che ne diamo non rimandano direttamente agli IIC, ma indicano gli IIC come il luogo finale dove convergono i fattori che definiscono il sistema socio-economico-culturale nel quale viene proposta l'offerta di formazione in italiano L2.

\subsection{GLI STUdenti DEI CORSI Di ITALIANO L2 ORganizzati DAGLI IIC}

Il primo dato quantitativo sul quale vorremmo richiamare l'attenzione è costituito dal numero di studenti che frequentano i corsi di italiano L2 organizzati dagli IIC, mettendolo a confronto con quello relativo al 1995. Secondo Italiano 2000, 45699 studenti hanno frequentato i corsi di italiano L2, mentre nel 1995 il loro numero era di 33065, con una differenza di +12634 studenti (+ 38,2\%). 


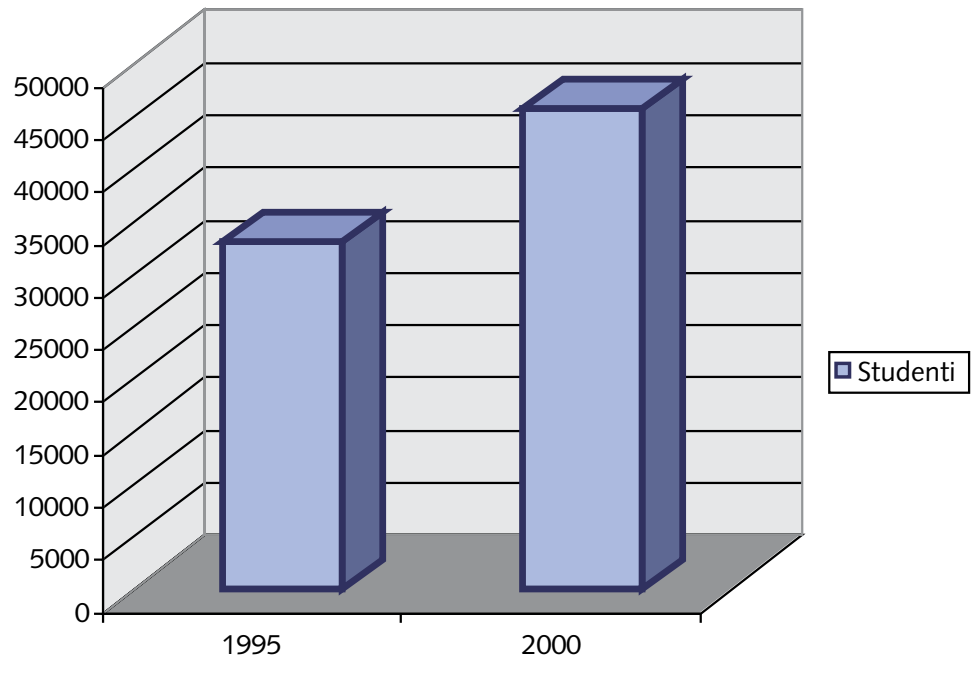

Grafico n. 1 - Andamento degli studenti iscritti ai corsi degli IIC

Già questo dato è di per sé molto interessante ${ }^{5}$ : negli ultimi cinque anni c'è stato un aumento consistente di affluenza di studenti ai corsi di italiano, e ciò significa che il sistema complessivo della lingua - cultura - società italiana, tramite l'offerta formativa messa in atto localmente dagli IIC, ha saputo rappresentare un fattore di attrazione che ha provocato una forte crescita. Alla luce di queste cifre, le linee lungo le quali si sono sviluppati complessivamente gli interventi hanno dato un risultato positivo. Dobbiamo chiederci quale sia il rapporto fra gli IIC e gli altri soggetti che localmente offrono formazione in italiano L2: non è da escludere a priori, infatti, la possibilità di una concorrenza fra i diversi soggetti che, in un sistema chiuso in equilibrio, può portare

545699 è il numero degli studenti che nell'anno 2000 hanno frequentato i corsi di italiano L2 presso gli IIC. Per un corretto confronto con i valori del 1995, si sono messi a confronto solo gli Istituti presenti sia nell'indagine del 1995 che in quella del 2000. il vantaggio a favore dell'uno, lo svantaggio per l'altro. Alla luce dei dati complessivi acquisiti dall'inchiesta e dalla fase di espansione del mercato delle lingue, la tendenza è generale e riguarda anche le altre agenzie culturali (il sistema delle scuole private di italiano, i corsi organizzati dai sistemi locali di educazione permanente, altre realtà italiane come ad esempio la Società Dante Alighieri).

I dati forniti dall'indagine della Società Dante Alighieri (1999) confermano il numero in aumento dei frequentanti i corsi di italiano L2; in diversi paesi le scuole private sono in aumento e comunque non registrano cali complessivi di studenti. Secondo Baker - Eversley (2000: 6), i corsi di italiano a Londra si collocano al quinto posto fra tutte le lingue (con soli tre corsi in meno del tedesco) ${ }^{6}$.

Rimaniamo sempre in area anglofona e passiamo agli U.S.A. La stima quantitativa più recente (Lebano, 1999) mostra che l'italiano è la quarta lingua più insegnata nella fascia alta del sistema di istruzione degli Stati Uniti, con una variazione in aumento dal 1995 al 1998 del 12,6\%, unica fra le prime quattro lingue negli U.S.A. a vedere una variazione in aumento ${ }^{7}$. Sempre Lebano (1999) ci fornisce un dato interessante: non sono più i discendenti dei nostri emigrati a costituite la maggioranza dei frequentanti i corsi di italiano, ma stranieri non di origine italiana: il $61 \%$ contro il 39\%. Si tratta di un'inversione di tendenza molto importante sulla quale torneremo in sede di interpretazione generale dei dati e di verifica delle ipotesi dell'indagine.

\footnotetext{
${ }^{6}$ Le lingue che hanno più corsi a Londra nell'anno scolastico 1999 - 2000 sono state l'inglese (497 corsi), il francese (474), lo spagnolo (389), il tedesco (279) e l'italiano (276). La ricognizione di Baker - Eversley (2000) prende in considerazione i corsi di lingua giornalieri e serali offerti a ogni livello da strutture pubbliche in Greater London.

Si assiste a un calo del francese $(-3 \%)$ e del tedesco $(-7 \%)$, e a una situazione di sostanziale stabilità dello spagnolo.
} 


\subsection{IL NUMERO DEI CORSI DI ITALIANO L2 ORGANIZZATI} DAGLI IIC

Nel 1995 i corsi organizzati sono stati 2346; nel 2000 sono stati 3684 , con un aumento di +1338 corsi $(+57 \%)$.

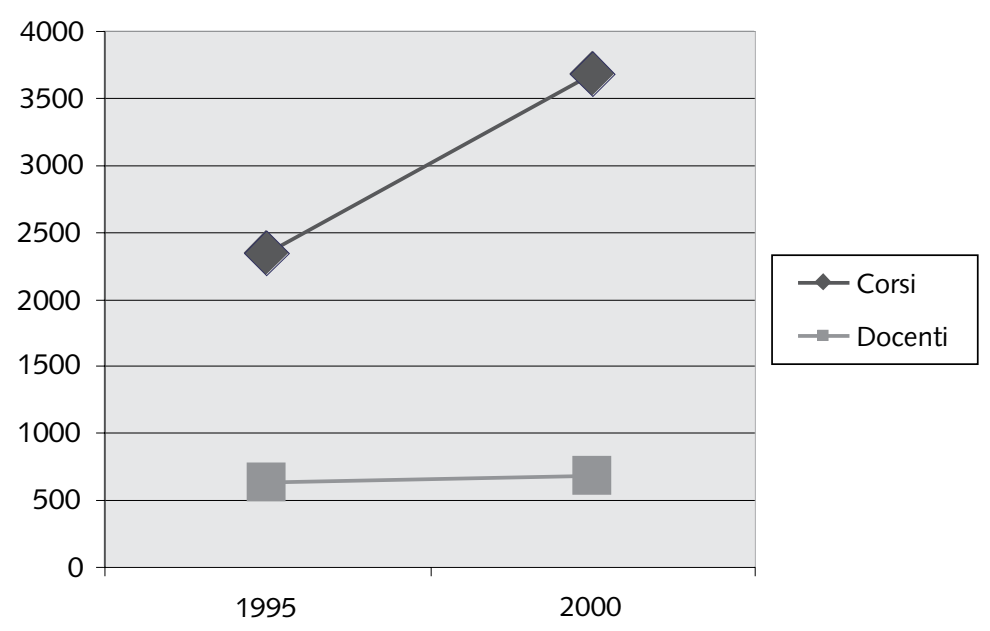

Grafico n. 2 - Andamento dei corsi e del numero dei docenti presso gli IIC

Tali cifre da un lato confermano la solidarietà dei legami fra il numero di studenti che si iscrive ai corsi e il numero dei corsi organizzati dagli IIC, ma indica anche lo svilupparsi di un rapporto tra numero studenti e numero corsi che appare favorevole agli studenti, i quali non vengono addensati nelle classi. Il numero di corsi aumenta percentualmente più del numero degli studenti e ciò implica che le classi aumentano avendo mediamente un numero minore di allievi, a tutto favore della correttezza della metodologia glottodidattica: o meglio: in presenza di condizioni favorevoli strutturalmente per la messa in atto di interventi corretti sul piano della metodologia glottodidattica.
Nel 1995, infatti, il rapporto studenti - corsi era di 14 studenti per corsi, cifra che si colloca entro i parametri di un corretto quadro di intervento glottodidattico. Nel 2000 tale rapporto scende a 12,4 studenti per corso, rendendo ancora più favorevole il contesto, che appare sempre più capace di accettare interventi vicini alle proposte glottodidattiche orientate verso la personalizzazione dei percorsi formativi e al lavoro per piccoli gruppi.

Le cifre presentate ci possono spingere, comunque, a fare l'ipotesi che proprio tali caratteri dell'offerta formativa costituiscano fattori che spingono alla scelta, da parte degli stranieri, dell'italiano piuttosto che di altre lingue, nel caso che queste si presentino con corsi più affollati e perciò con situazioni didattiche meno favorevoli contestualmente all'apprendente. Se teniamo presente, inoltre, il grande sforzo di aggiornamento che ha caratterizzato il generale sistema della formazione in Italia e la sua capacità di riverberarsi sull'italiano L2, tramite i docenti e gli altri elementi costitutivi dell'offerta formativa, appare ancor più fondata l'idea dell'attrattività glottodidattica della nostra lingua non tanto a causa della caratterizzazione tecnologica delle strutture, quanto per la qualità dell'impegno glottodidattico messo in atto dalle strutture e dai docenti a favore dell'apprendente.

\subsection{DOCENTI DEI CORSI DI ITALIANO L2 ORGANIZZATI DAGLI IIC}

Nel 1995 i docenti impegnati negli IIC erano 624; nel 2000 sono stati 686 (dati paragonabili; in assoluto 715), con un aumento di 62 unità (+ 9\%, ma in assoluto $+12,7 \%)$. Rispetto ai considerevoli aumenti degli studenti e del numero dei corsi, i docenti aumentano in misura molto più contenuta. Questo fa sì che la situazione sia caratterizzata dall'aumento delle ore di insegnamento alle quali sono chiamati i docenti. 
5.5 I DATI RELATIVI A STUDENTI, CORSI E DOCENTI DAL PIANO GENERALE ALLE REALTÀ LOCALI

Su 60 IIC dei quali abbiamo i dati, dal 1995 al 200028 IIC (pari al 46.6\%) hanno visto diminuire il numero dei loro docenti; 1'11,6\% ha mantenuto le stesse quote; 26 IIC (pari al 43,3\%) hanno aumentato i docenti. La situazione, pertanto, non è semplicemente assimilabile alla tendenza all'aumento, pur se limitato.

Il dato sui docenti fa correlazione con quello relativo al numero dei corsi e degli studenti: 22 IIC $(36,6 \%)$ hanno diminuito il numero dei propri corsi, ma 36 (60\%) lo hanno aumentato. Parallelamente, 24 IIC (40\%) hanno diminuito gli studenti, contro $36(58,3 \%)$ che vedono aumentare il loro numero. La perdita di studenti ha superato le 6000 unità.

Si può dire, allora, che la tendenza messa in mostra dal dato complessivo, nettamente orientato verso l'aumento degli studenti, dei corsi e, in misura minore, dei docenti non riguarda tutti gli IIC. Ci sono realtà in cui diminuiscono docenti, corsi e studenti. La diminuzione dei docenti è più ampia di quella dei corsi / degli studenti: la differenza fra le due dimensioni ci fa capire l'area in cui la diminuzione dei docenti è frutto di una razionalizzazione della struttura, pur in presenza di un mercato in aumento. Negli altri casi, la diminuzione degli studenti (se non è conseguenza del fatto che diminuiscono o non ci sono i corsi) porta a far calare (se non addirittura a chiudere completamente) i corsi stessi, e ovviamente a interrompere i rapporti fra gli IIC e i docenti.

\subsection{UN'IPOTESI INTERPRETATIVA}

Questi risultati messi in luce da Italiano 2000 ci spingono a qualificare gli ultimi anni del Novecento come segnati da una netta tendenza all'aumento della richiesta di italiano L2 nel mondo. Le cause di tale andamento positivo possono essere trovate nell'interazione fra la dimensione di tipo linguistico e altre dimensioni: quella economico-produttiva, quella socioculturale e delle immagini del paese e della sua società, quella della gestione delle politiche culturali e formative messe in atto dalle nostre istituzioni e dagli IIC in particolare. Il complessivo 'sistema Italia' sembra funzionare presso gli stranieri, nel senso che diventa sempre più presente negli altri paesi, non rimanendo estranea a tale presenza la dimensione della potenziale emigrazione verso l'Italia.

Possiamo dire, per valutare complessivamente le cause di tale situazione, che negli ultimi cinque anni si sia consolidato un nesso fra la tradizionale forza di attrazione della nostra lingua e i nuovi fattori economico-sociali-culturali: tale nesso fa sì che l'Italia e l'italiano siano sempre più presenti come punti di riferimento nelle scelte che gli stranieri fanno circa gli investimenti formativi nel campo linguistico.

La realtà della diffusione dell'offerta di formazione in italiano L2, se esaminata più da vicino, ci sembra attraversata da una frattura netta: un quarto degli IIC vede diminuire gli studenti. Più di un terzo diminuisce il numero dei propri corsi. Il 45\% diminuisce il numero dei docenti, con evidenti riflessi sulle possibilità di occupazione di chi, in Italia e all'estero, si forma per diventare docente di italiano L2.

Ci si presenta evidente una situazione attraversata nettamente da una frattura: una parte appare in regresso, una parte in forte crescita. Il dato iniziale, in altri termini, è ingannevole, perché può far credere che in tutto il mondo, in tutta la rete degli IIC si manifesti una tendenza positiva all'aumento degli studenti: invece la situazione è variabile, la superficie del mondo linguistico dell'italiano L2 è corrugata, l'aumento di un terzo del numero degli studenti iscritti ai corsi è solo una misura media. Il dato generale che abbiamo mostrato per primo, cioè che il numero degli studenti dal 1995 al 2000 è aumentato di un terzo, in realtà sarebbe stato ancora 
più positivo se gli IIC che hanno visto perdere studenti avessero almeno mantenuto le loro posizioni.

Immaginiamo una realtà virtuale: come sarebbe la situazione dell'italiano nel mondo se non ci fosse stata la perdita di studenti in un certo numero di IIC, e quindi se la tendenza positiva si fosse distribuita omogeneamente in tutto il mondo. Il grafico n. 3 mostra la realtà virtuale dell'italiano L2 se non ci fosse stata la perdita dei più di 6.000 studenti in 24 IIC.

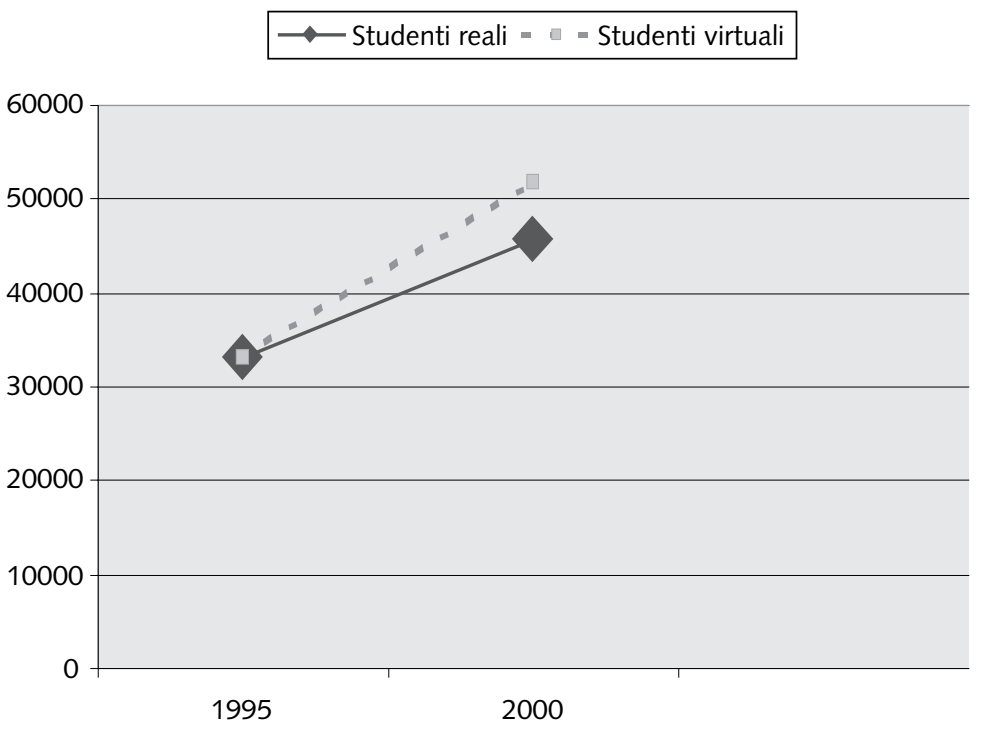

Grafico n. 3 - Dati reali e dati virtuali sul numero di iscritti ai corsi degli IIC

Non ci sembra possibile proporre un'interpretazione dei complessi e contraddittori processi che riguardano l'italiano diffuso fra gli stranieri, anche solo limitando l'analisi ai dati più macroscopici, senza ritornare alle caratteristiche di quel 'mercato delle lingue' che è, in realtà, metafora del sistema globalizzato e insieme in competizione di modelli di economia e di sistemi economici, di culture e di identità culturali, di modi di vivere e di società, di valori individuali e collettivi.

Dal punto di vista del mercato delle lingue, il numero degli studenti iscritti ai corsi di italiano nel mondo non può certo essere paragonato a quello dell'inglese, del francese, del tedesco, dello spagnolo. La graduatoria, però, non è statica: la stessa supremazia dell'inglese dipende fortemente dal suo valore d'uso strumentale e immediato nella comunicazione quotidiana e nelle interazioni di affari. Pur essendo una lingua di grande spessore culturale, la sua attrattività non dipende oggi solo da questa caratteristica. Ciò significa che il valore d'uso della prima lingua a diffusione internazionale negli scambi interlinguistici dipende dalle funzioni che le assegnano i suoi apprendenti: ne derivano implicazioni che, a partire dalla gamma di funzioni per le quali l'inglese è usato, investono le sue caratteristiche strutturali. La riduzione semplificante, sempre più lamentata per l'inglese nel momento in cui si diffonde in modo egemone, deriva da una spinta in tal senso da parte dei suoi nuovi apprendenti non nativi. Le conseguenze di tali processi, anche nei rapporti di concorrenza con le altre lingue, non sono semplici né immediatamente identificabili: non è da escludersi, in futuro, un riposizionamento stesso dell'inglese nella graduatoria delle lingue più diffuse internazionalmente proprio a causa delle ristrutturazioni interne dovute alle pressioni dei suoi apprendenti non nativi. Le lingue cambiano nell'uso: la struttura interna ne è coinvolta, ma questa interagisce anche sui valori che decidono l'adozione o meno di un idioma nello scambio interlinguistico.

L'italiano si colloca in una situazione di competizione con alcune lingue in determinate realtà locali: il francese, il tedesco, lo spagnolo possono precedere l'italiano come oggetto di apprendimento se consideriamo la questione secondo una visione generale, ma se la consideriamo più analiticamente, l'italiano riesce a entrare in competizione con tali lingue in 
diverse situazioni locali. La competitività dell'italiano in tali realtà viene a dipendere da fattori di contesto, da processi di tipo economico-commerciale, dalla capacità di presa dei modelli linguistico-culturali-sociali italiani su una determinata società. Può essere allora la presunta vicinanza fra modelli o il fatto che sia punto di riferimento negli scambi economici a orientare sulla nostra lingua un numero consistente di apprendenti e a posizionare l'italiano in seria concorrenzialità con lingue generalmente più diffuse nel mondo.

Se accettiamo l'idea di una competitività generale entro il mercato delle lingue, riconosciamo che gli elementi di variabilità caratterizzano tale sistema: ciò che sembra specifico, come elemento negativo, della condizione dell'italiano diffuso fra gli stranieri, appare in realtà un tratto generale dei rapporti contemporanei fra le lingue. Da questo punto di vista, possiamo parlare, allora, di una interpretazione italiana di tale variabilità, non di un tratto esclusivo della nostra condizione. Da ciò una conseguenza: in ciò che vi è di positivo e di negativo nei tratti di variabilità generalizzata all'intero sistema del mercato delle lingue, l'italiano vi contribuisce e ne risente.

Appare plausibile, allora, ritornare al numero in aumento degli studenti stranieri di italiano dal 1995 al 2000: è frutto sicuramente degli interventi del sistema formativo, culturale, sociale, economico, istituzionale italiano. Esso è però anche conseguenza dell'esistenza del mercato mondiale delle lingue, delle sue tendenze massificanti e globalizzanti da un lato, variazioniste e localiste dall'altro. Siamo in un momento storico in cui la diffusione dell'apprendimento delle lingue è in fase di forte espansione, trovandosi a essere campo di dialettica fra vari sistemi nazionali, ma essendo anche un settore di produzione economica. Si parla di industria delle lingue come di un settore della produzione economica applicata al campo culturale, coinvolgente l'elaborazione tecnologica, l'efficienza dei sistemi formativi, le capacità di elaborazione intellettuale, di ricerca scientifica, di innovazione.
Paradossalmente, l'immane impegno messo dai rispettivi paesi nella diffusione dell'inglese e del francese ha comportato un effetto di trascinamento sull'intero sistema: in un mercato mondiale delle lingue che è in espansione anche l'italiano si trova ad aumentare il numero degli stranieri che vi si avvicinano per apprenderlo. E non è importante che per essi sia la seconda, la terza o la quarta L2 appresa: è rilevante che il processo coinvolga in modo sistematico la nostra lingua.

Con tale affermazione non vogliamo sminuire il ruolo delle azioni positive di propulsione messe in atto in questi ultimi decenni per diffondere l'italiano e i loro effetti di stimolo $^{8}$. Non vogliamo nemmeno giustificare l'assenza di interventi sulla base del fatto che il mercato trascinerebbe anche la nostra lingua all'aumento della sua diffusione pur in assenza di grandi investimenti. Vogliamo in realtà sottolineare due aspetti che si aggiungono alle azioni positive che hanno consentito di aumentare il bacino di utenza della nostra lingua.

Innanzitutto, in un mercato in aumento, e in un mercato che si basa sulla variabilità dei processi, appare ingiustificabile la perdita di pubblico reale manifestatasi in modo consistente in parecchi IIC. Se la perdita di studenti si fosse

${ }^{8}$ Si pensi a quanto fanno le Università per Stranieri di Siena e di Perugia, alla diffusione delle certificazioni CILS - Certificazione di Italiano come Lingua Straniera di Siena, CELI di Perugia, IT della Terza Università di Roma, di quella della Società Dante Alighieri, delle diverse certificazioni elaborate localmente da altre agenzie culturali. Si pensi al sistema delle scuole private di italiano e alla loro capacità di penetrare nei paesi stranieri e di attirare studenti in Italia. Non si dimentichi, infine, quanto il sistema scolastico istituzionale italiano produce in termini di formazione linguistica del 1.700.000 immigrati stranieri adulti e dei 140.000 bambini presenti nella scuola; e quanto nella stessa direzione fanno le agenzie del volontariato. A ciò si aggiunga la rete delle università straniere. E non da ultimo come importanza, le attività delle nostre scuole all'estero, dei corsi di lingua e cultura, gli interventi culturali promossi dalle nostre istituzioni all'estero. 
svolta entro un processo controbilanciato solo dalle azioni positive delle istituzioni italiane, sarebbe stata valutata meno gravemente rispetto a una considerazione che vede l'intero sistema muoversi verso l'espansione. Ciò ci spinge a sottolineare un rischio che riteniamo gravissimo: in una situazione di variabilità e di fluttuazione poco controllabile, la perdita di studenti, cioè di pubblico reale, può essere non grave se non viene intaccata la fascia di pubblico potenziale dell'italiano L2. In un mercato in espansione, però, la perdita di pubblico reale in situazioni dove le condizioni potrebbero agire tutte in direzione opposta significa correre il rischio di contribuire a creare una fascia di non-pubblico, cioè di soggetti per i quali non solo e non tanto la lingua italiana non rientra nei progetti di investimento formativo, ma la stessa Italia, i suoi tratti economici, sociali, culturali escono fuori da ogni possibilità di contatto. Le conseguenze dirette, in questo caso, non sono solo sulla lingua italiana, ma sull'intero 'sistema Italia'.

Il secondo elemento che vogliamo sottolineare è costituito dal fatto che il mercato delle lingue non si concretizza solo in processi di concorrenza. L'italiano può e deve trovare appoggi e alleanze con altre lingue, di volta in volta scegliendo le funzioni e gli obiettivi di tali alleanze: penetrare in un determinato mercato, evitare il soffocamento a fronte di un'azione di diffusione massiccia di un'altra lingua, partecipare alla costruzione di un'identità che supera quella nazionale.

Quest'ultimo punto investe direttamente la nostra posizione entro il sistema europeo delle lingue. A nostro avviso, il mercato mondiale delle lingue è in espansione grazie al contributo determinante (pur se non esclusivo) della politica linguistica delle istituzioni europee: dai progetti dei Livelli Soglia al recente Framework comune per la diffusione delle lingue, dalla tutela delle lingue meno diffuse ai modelli interculturali di plurilinguismo, il baricentro dell'elaborazione glottodidattica e della progettualità orientata allo sviluppo di un'idea di cittadinanza europea fondata sul contatto fra le lingue e sul loro apprendimento si è sempre più determinato nel vecchio continente.

Ne è derivata anche per l'italiano la condivisione di una linea che ha avuto come effetto quello di renderci partecipi della grande spinta propulsiva derivante dall'impegno teorico-metodologico e di investimento di risorse maturatosi a livello comunitario.

Anche solo tenendo conto di tale condivisa identità europea, appare ancor più vasto e aperto il campo entro il quale può svolgersi l'azione istituzionale di diffusione della nostra lingua, tesa a rendere pubblico reale quello che è solo pubblico potenziale del contatto con la nostra lingua e cultura; e altresì appare ugualmente centrale l'obiettivo di conquistare fasce sempre più vaste di quello che si trova nella condizione di non-pubblico.

\section{NuOvi SVILUPPI}

Italiano 2000 ha messo in evidenza la netta espansione dell'italiano come lingua oggetto di apprendimento nel mondo: una tendenza che globalmente è continuata anche dopo il 2000, come confermano i dati statistici più recenti del Ministero degli Affari Esteri. Questo risultato positivo è bilanciato, però, dalla fragilità di tale processo: la forte variabilità dei modi di diffusione della nostra lingua implica la sua forte dipendenza da fattori di contesto che possono facilmente mutare da positivi in negativi, facendo regredire le posizioni conquistate. Lasciata la diffusione dell'italiano sostanzialmente al libero gioco del mercato delle lingue e sostenuta da risorse non comparabili con quelle messe in gioco da altre realtà statali, la nostra lingua rischia di vedere limitato il suo potenziale di diffusione: l'adattabilità di un sistema di offerta formativa 'a geometria variabile' rischia di trasfor- 
marsi nella dispersione delle esperienze dovuta a diverse cause, fra le quali vanno annoverate, ad esempio, le conseguenze del legame con il nostro sistema delle imprese multinazionali, nel momento in cui, come oggi, viva una fase problematica.

Un mercato delle lingue in espansione mondiale, trascinato dalla planetarizzazione dell'inglese, fa aumentare gli studenti e i corsi di lingua straniera: di tutte le lingue, anche dell'italiano. La mondializzazione, intesa dalla parte della infinitamente aumentata possibilità di incontro fra le genti, ha creato le condizioni per il contatto intenso e continuo fra gli idiomi, sviluppando la spinta alla loro conoscenza. Mondializzazione e glocalizzazione, planetarizzazione e mantenimento delle identità locali si sostengono reciprocamente e costituiscono la condizione del mercato mondiale delle lingue in espansione. La diffusione crescente dell'italiano ha le sue prime radici in questa situazione mondiale.

Il senso del nostro discorso è ben chiaro: confidare nella rendita di capitale costituita dal legame fra la lingua italiana e la nostra tradizione storico-culturale non basta per competere nell'attuale fase di rapporto fra i sistemi lingua-cultura-società-economia. Non ci si possono permettere, allora, a nessun livello, trionfalismi senza fondamento né cupi pessimismi.

Per ciò che concerne l'identità e il destino internazionale dell'italiano la ricerca scientifica non può indulgere ad acritiche visioni trionfalistiche né tanto meno sciogliersi nei lai per un destino avverso, né, infine, la ricerca scientifica può trasformare i propri risultati in illecebra al potere politico. Indagini come Italiano 2000 possono avere tempi lunghi quanto a uso dei risultati, come già accadde all'inchiesta di Baldelli. L'esigenza di stare entro il mercato mondiale delle lingue (o, se si preferisce, nel nuovo ordine linguistico globale: Maurais, 2003) impone di avere, al contrario, dati sempre aggiornati e affidabili, per poterli utilizzare nella elabo- razione di politiche culturali per la diffusione della lingua in risposta alle sempre variabili esigenze di formazione linguistica emergenti nel mondo globalizzato.

Già Baker, Eversley (2000) hanno sottolineato quanto il nuovo volto plurilingue delle capitali del 'primo mondo' debbano ai processi economici, e quanto dalle politiche di diffusione delle lingue si possano giovare i sistemi economico-produttivi9. In Italiano 2000 avevamo elaborato la provocatoria ipotesi che la lingua italiana aprisse la strada alla nostra economia nel mondo. Ora, grazie al rapporto ICE, l'economia italiana ci disvela la sua attuale identità: pensare di ignorarla nel progettare linee di sostegno alla diffusione dell'italiano è per lo meno ingenuo, se non addirittura dannoso per la lingua e per l'economia. Se davvero la lingua italiana ha un ruolo nella diffusione dell'economia, sta ora a chi si occupa di lingua assumersi la responsabilità di sviluppare una adeguata azione che rivalorizzi i tratti positivi della nostra identità linguistico-culturale creando in tal modo le condizioni affinché questi passino alla dimensione economica: la qualità, la creatività, i valori estetici, l'espressività, la polifonicità delle voci nell'unità si dovranno ancora legare alle forme solari di vita, alla generosità nell'accoglienza, alla capacità di produzione e diffusione di prodotti ad alta concentrazione degli elementi positivi della nostra identità, che gli stranieri attendono. Non ci si aspetti di veder competere la nostra lingua con l'inglese: il nostro idioma è visto dagli stranieri come alternativa non tanto allo strumentalmente planetario inglese, ma come forma simbolica alternativa nella ricerca di nuovi significati nel contatto con le altre culture, significati che si vedono incarnati anche in un sistema di vita, sociale e produttivo, ovvero il 'sistema Italia'. Di conseguenza, interrogarsi su quale sia oggi la situazione dell'italiano

9 Per gli studi sui nuovi panorami plurilingui delle città italiane ved. Bombi \& Fusco (2005), Bagna \& Barni (2005a, 2005b, in stampa). 
nel mondo implica necessariamente fare ricorso a quanto avviene su altre dimensioni, prima fra tutte quella dove si situano i processi economico-produttivi di internazionalizzazione.

Tutto questo ha bisogno di nuova conoscenza, di nuovi dati: riteniamo che sia giunto il momento di pensare a un Italiano 2006.

\section{BIBLIOGRAFIA}

Baker Ph., Eversley J., 2000, Multilingual Capital, London, Battlebridge Publications.

BALDELli I., 1987, La lingua italiana nel mondo. Indagine sulle motivazioni allo studio dell'italiano, Roma, Istituto per l'Enciclopedia Italiana.

Bandini A., Barni M., Sprugnoli L., 1999, La CILS - Certificazione di Italiano come Lingua Straniera. In: M. Vedovelli (a cura di), Indagini sociolinguistiche nella scuola e nella società italiana in evoluzione, Milano, Franco Angeli: 407-419.

Calvet L.-J. 2002, Le marché aux langues, Paris, Plon.

Consiglio D'Europa, 1997-2001, Common European Framework of reference for languages. Learning, teaching, assessment, Strasbourg - Cambridge, Council of Europe - Cambridge University Press (trad. it. Quadro comune europeo di riferimento per le lingue. Apprendimento, insegnamento, valutazione, Scandicci - Firenze, La Nuova Italia, 2002).

Covino Bisaccia M.A., 1989, Motivazioni allo studio dell'italiano nei discenti stranieri presso l'Università per Stranieri di Perugia nell'anno accademico 1988. Analisi dei questionari B, Perugia, Guerra.

Covino Bisaccia M.A., 1990, Motivazioni allo studio dell'italiano nei discenti stranieri presso l'Università per Stranieri di Perugia nell'anno accademico 1988. Analisi dei questionari $A$, Perugia, Guerra

De Mauro T., Vedovelli M., 1996, La diffusione dell'italiano nel mondo e le vie dell'italianizzazione: problemi istituzionali e sociolinguistici. In: G. Tassello, M. Vedovelli (a cura di), Scuola, lingua e cultura nell'emigrazione italiana all'estero. Bibliografia generale (1970-1995), Roma, Centro Studi Emigrazione: 7-41.
ESPERIENZE E PROPOSTE, 1979, L'immigrazione straniera in Italia, Roma, CGIL.

Giacalone Ramat A. (a cura di), 1986, L'apprendimento spontaneo di una seconda lingua, Bologna, Il Mulino.

Lebano E., 1999, Survey on the italian language in the U.S.A., Welland, Ontario (CA), Soleil.

Maurais J., 2003, Towards a new linguistic order? in Maurais, Jacques \& Michael A. Morris (eds.), Languages in a globalising world, Cambridge, Cambridge University Press.

Maggini M., Parigi V., 1983, Italiana per tutti. "Italiano e oltre”, 3: 132 -135 .

Ministero degli Affari Esteri, Direzione Generale delle Relazioni Culturali, 1996, La promozione della cultura italiana all'estero, Roma, I.P.Z.S.

Società Dante Alighieri, 1999, Vivere italiano: il futuro della lingua. Bozza del rapporto dell'indagine svolta in collaborazione con il CNEL, presentato il 30 marzo 1999, Roma.

Vedovelli M., 1999, Le identità dell'italiano diffuso fra stranieri. In: S. Bruni (a cura di), Atti del corso di formazione iniziale per il personale della scuola da destinare all'estero, Siena, 22-26 luglio 1997, Siena, Università per Stranieri di Siena.

Vedovelli M., 2002a, Guida all'italiano per stranieri. La prospettiva del Quadro comune europeo per le lingue, Roma, Carocci.

Vedovelli M., 2002b, L'italiano degli stranieri. Storia, attualità, prospettive, Roma, Carocci.

Vedovelli, M., 2005, L'italiano nel mondo da lingua straniera a lingua identitaria: il caso freddoccino, in "SILTA - Studi Italiani di Linguistica Teorica e Applicata”, XXXIV, 2005, 3: 585-609.

Vedovelli M., Barni M., Miraglia L. (a cura di), 2001, Studio di fattibilità per una ricerca sulle motivazioni e sulle caratteristiche dei pubblici dei corsi di lingua e cultura italiana all'estero (Attività linguistico-culturali istituite ai sensi del D.L. 297/94 art. 636), rapporto di ricerca per il Ministero degli Affari Esteri, non pubblicato, Centro CILS, Siena, Università per Stranieri di Siena. 